\title{
Comparison of the prevalence of respiratory viruses in patients with acute respiratory infections at different hospital settings in North China, 2012-2015
}

Jianxing $\mathrm{Yu}^{1 \dagger}$, Zhengde Xie ${ }^{2 \dagger}$, Tiegang Zhang ${ }^{3 \dagger}$, Yanqin $\mathrm{Lu}^{4 \dagger}$, Hongwei Fan ${ }^{5 \dagger}$, Donghong Yang ${ }^{6 \dagger}$, Thomas Bénet ${ }^{7,8}$, Philippe Vanhems ${ }^{7,8,9}$, Kunling Shen ${ }^{2}$, Fang Huang ${ }^{3}$, Jinxiang Han ${ }^{4}$, Taisheng Li ${ }^{5}$, Zhancheng Gao ${ }^{6}$, Lili Ren ${ }^{*}$ and Jianwei Wang ${ }^{1 *}$

\begin{abstract}
Background: Acute respiratory infections (ARIs) are a great public health challenge globally. The prevalence of respiratory viruses in patients with ARIs attending at different hospital settings is fully undetermined.

Methods: Laboratory-based surveillance for ARIs was conducted at inpatient and outpatient settings of 11 hospitals in North China. The first 2-5 patients with ARIs were recruited in each hospital weekly from 2012 through 2015. The presence of respiratory viruses was screened by PCR assays. The prevalence of respiratory viruses was determined and compared between patients at different hospital settings.

Results: A total of 3487 hospitalized cases and 6437 outpatients/Emergency Department (ED) patients were enrolled. The most commonly detected viruses in the hospitalized cases were respiratory syncytial virus (RSV, 33.3\%) in children less than two years old, adenoviruses (13.0\%) in patients 15-34 years old, and influenza viruses (IFVs, 9.6\%) in patients $\geq 65$ years. IFVs were the most common virus in outpatient/ED patients across all age groups (22.7\%). After controlling for the confounders caused by other viruses and covariates, adenoviruses (adjusted odds ratio [aOR]: 3.97, 99\% confidence interval [99\% Cl]: 2.19-7.20) and RSV (aOR: 2.04, 99\% Cl: 1.34-3.11) were independently associated with increased hospitalization in children, as well as adenoviruses in adults (aOR: 2.14, 99\% Cl: 1.19-3.85). Additionally, co-infection of RSV with IFVs was associated with increased hospitalization in children (aOR: 12.20, 99\% Cl: 2.65-56.18).
\end{abstract}

Conclusions: A substantial proportion of ARIs was associated with respiratory viruses in North China. RSV, adenoviruses, and co-infection of RSV and IFVs were more frequent in hospitalized children (or adenoviruses in adults), which might predict the severity of ARIs. Attending clinicians should be more vigilant of these infections.

Keywords: Respiratory tract infections, Viruses, Risk factors, Hospitalization, Sentinel surveillance

\footnotetext{
* Correspondence: renliliipb@163.com; wangjw28@163.com

${ }^{\dagger}$ Equal contributors

${ }^{1} \mathrm{MOH}$ Key Laboratory of Systems Biology of Pathogens and Christophe

Mérieux Laboratory, IPB, CAMS-Fondation Mérieux, Institute of Pathogen

Biology (IPB), Chinese Academy of Medical Sciences (CAMS) \& Peking Union

Medical College, Beijing 100730, People's Republic of China

Full list of author information is available at the end of the article
} 


\section{Background}

Acute respiratory infections (ARIs) are a major global public health problem because of their high morbidity and mortality [1]. They represented 18.8 billion upper respiratory tract infections (URTIs), 150 million lower respiratory tract infections (LRTIs) and 2.6 million deaths in the global burden of diseases estimates in 2013 $[2,3]$. In a substantial proportion of patients, ARIs are found to be associated with respiratory viruses (RVs) $[1,4-6]$. In addition to their high frequency, infection with RVs can lead to severe outcomes, including hospitalization and death [1]. Notably, respiratory syncytial virus (RSV) causes most of the severe LRTIs in young children [7], while influenza viruses (IFVs) and human rhinoviruses (HRVs) are the predominant causative agents in hospitalized adults with pneumonia [8].

Shortly after the SARS events in 2002 [9], regional and nationwide laboratory-based surveillance studies for ARIs were conducted in China to help clarify the epidemiological feature of RVs by employing the highly sensitive modern molecular techniques, such as polymerase chain reaction (PCR) [10-16]. These studies are very helpful for characterizing the prevalence and burden of specific RVs in pre-vaccine Chinese populations (i.e., vaccines against Streptococcus pneumoniae, Haemophilus influenzae type b, and influenza viruses are currently not incorporated into the national immunization program [17]). In addition, these studies are useful to identify new emerging viral infections [18-21]. However, few previous studies have studied RVs across different hospital settings (e.g., outpatient, emergency room, inpatient wards and intensive care units) and explored etiological, environmental and host factors that predisposed patients to severe disease presentation, such as hospitalization or intensive care admission. It is very important to clarify the most common viral agents causing ARIs and their relationship with the severe disease presentation for clinicians who treated their patients with ARIs at different hospital settings.

The purpose of the study was to determine the prevalence of RVs in patients with ARIs at different hospital settings and to identify factors that were most likely associated with hospitalization due to ARIs.

\section{Methods}

\section{Patient enrollment}

The patients were enrolled from January 1, 2012 through December 31, 2015 at 11 hospitals (Additional file 1) in North China according to the following criteria: (1) had symptoms of acute infection, defined as fever (a body temperature $>38.0{ }^{\circ} \mathrm{C}$ ) or hypothermia (a body temperature $<35.5{ }^{\circ} \mathrm{C}$ ), chill, or leukocytosis (a white blood cell count $>10,000 / \mathrm{ml}$ ) or leukopenia (a white blood cell count $<4000 / \mathrm{ml}$ ); (2) had signs/symptoms of acute respiratory illness, defined as sore throat, runny nose, cough, sputum-production, shortness of breath, wheezing or crackles, or chest pain; and (3) with or without radiograph abnormality.

Patients were screened for enrollment eligibility by attending physicians of the 11 hospitals in the following settings: emergency department (ED), outpatient (internal medicine, pediatric, respiratory or febrile illness clinics) and/or inpatient (the respiratory medicine ward or the intensive care unit). The first two to five eligible patients (children and adults) in each hospital were enrolled each week via convenience sampling. Enrollment was conducted simultaneously in both inpatient and outpatient/ED settings in four hospitals. Five hospitals enrolled patients in outpatient/ED settings and two hospitals enrolled patients in inpatient settings. To avoid duplicated inclusion, patients who were referred from other hospitals or not initially diagnosed in our 11 hospitals were excluded. The study is observational and did not intervene with the choice of clinical management, namely hospitalization or ambulatory care.

\section{Data and specimen collection}

Respiratory specimens (nasopharyngeal swab or aspirate, and sputum), regardless of the clinical severity, were regularly collected by physicians as quick as possible after patient enrollment. Bronchoalveolar lavage or pleural puncture fluid were collected upon physicians' orders. The collected respiratory specimens were stored immediately in viral transportation media (VTM, Copan, Brescia, Italy) at $-70{ }^{\circ} \mathrm{C}$ at the hospitals before being transported to the central laboratory for viral screening. Demographic and clinical information were collected from each enrolled patient via a standardized case reporting form.

\section{Laboratory testing}

Total nucleic acids (DNA and RNA) were extracted from respiratory specimens using RNA/DNA mini-kits (Qiagen, Valencia, CA) or Nucliens easMAG (bioMérieux, Marcy I' Etoile, France), according to the manufacturers' instructions. The presence of IFVs (A, B and C), HRVs, parainfluenza viruses (PIVs 1-4), RSV (A and B), human adenoviruses (HAdVs), human bocaviruses (HBoV), human metapneumovirus (hMPV), and human coronaviruses (HCoV-229E, OC43, NL63 and HKU1) were screened by reverse-transcriptase polymerase chain reaction (RT-PCR) and PCR assays as described previously [10].

\section{Statistical analysis}

The prevalence (or detection rate) of viruses was calculated by dividing the number of positive cases by the total case numbers tested for that virus. The 95\% binomial confidence interval $(95 \% \mathrm{CI})$ for detection rates 
were calculated by the Pearson-Klopper method. To compare variables between subgroups of patients, we used Chi-square tests or Fisher's exact tests for categorical variables and Wilcoxon rank-sum or Kruskal-Wallis tests for continuous variables as appropriate. Two-sided $p$ value of $<0.05$ was considered statistically significant.

To explore factors that predispose patients to hospitalization, multivariable logistic-regression modeling was used. The dependent variable was hospitalization (hospitalized $=1$, outpatient $/$ ED patient $=$ $0)$. The independent variables were the presence or absence of specific viral pathogen (identified $=1$, unidentified $=0$ ). To assess and quantify the contribution of a specific virus without regard to the presence of other pathogens, this model includes all tested respiratory viruses as well as their two-way interactions. In this case, the effect of co-infection upon hospitalization of ARIs, assigned as interaction term of pairs of viruses, could be explained as the excessive increment/decrement of hospitalization that could not be explained by additive effects or confounding of either composite virus. Variables (e.g., age, gender, surveillance year, and season of illness onset), were also included in multivariable model to account for potential confounding if significant at $p<$ 0.10 in bivariate analysis. Since we hypothesized that the prevalence of RVs was disproportionately affected by the age of patients, multivariable models were constructed for patients of all ages, children aged $0-14$ years, adults aged 15-64 years and elderly aged $\geq 65$ years. The results of multivariable analyses were exhibited as adjusted odds ratio (aOR), namely the exponential of model coefficients. As we included a large number of cross-product terms in our model, type I errors and over-fitting will be significant problems. We used 99\% CI for aORs instead in the multivariable analyses. All statistical analyses were conducted using $R$ version 2.15.3 (R Foundation for Statistical Computing, Vienna, Austria) [22].

\section{Results}

\section{Demographic and clinical characteristics}

In total, 9924 patients were enrolled from the 11 hospitals. Of these patients, 3487 (35.1\%) were hospitalized cases and $6437(64.9 \%)$ were outpatient/ED patients. The median age of hospitalized cases was 8.8 years (interquartile range [IQR]: $3.0-52.0$ years), which was much lower than the median age of outpatient/ED patients (28.2 years, IQR: $15.6-42.1$ years $)(p<0.001)$. Compared with outpatient/ED patients, the hospitalized cases were more frequently male $(58.3 \%$ vs. $51.7 \%$, p < $0.001)$, children less than five years old $(34.6 \%$ vs. $12.3 \%$, $\mathrm{p}<0.001)$ and elderly patients $\geq 65$ years $(17.3 \%$ vs. $6.3 \%$, $\mathrm{p}<0.001$ ) (Table 1).

Of all patients, $5230(52.7 \%)$ were URTIs and 4694 (47.3\%) were LRTIs. Compared with outpatient/ED patients, the hospitalized cases were diagnosed more often with LRTIs $(95.4 \%$ vs. $21.3 \%, p<0.001)$ and pneumonia $(88.2 \%$ vs. $17.6 \%, \mathrm{p}<0.001)$, but less often with URTIs $(4.6 \%$ vs. $78.7 \%, p<0.001)$. Moreover, some of clinical sign/symptoms were more frequent among the hospitalized cases than the outpatient/ED patients, including cough, wheezing/crackles, tachypnea, dyspnea, anemia, and leukocytosis ( $\mathrm{p}<0.001$ for each symptom). In contrast, fever of $>38.5^{\circ} \mathrm{C}$, malaise, headache, rhinorrhea, and sore throat ( $\mathrm{p}<0.001$ for each symptom) were more frequent among the outpatient/ED patients.

\section{Prevalence of viruses}

At least one virus was detected in 1369 (39.3\%) hospitalized cases and 2459 (38.2\%) outpatient/ED patients ( $p=$ 0.31 ). The overall detection rates of RVs varied disproportionately according to age of patients and hospital settings. The highest rates of RV infection were in children under two years of age $(72.6 \%$ and $47.1 \%$ in hospitalized and outpatient/ED patients, respectively; $\mathrm{p}<$ 0.001 ) and the lowest rates of RV infection were in patients $\geq 65$ years $(22.9 \%$ and $35.1 \%$ in hospitalized and outpatient/ED patients, respectively; $\mathrm{p}<0.001)$ (Table 2). The prevalence of specific RVs was significantly higher $(p<0.05)$ in hospitalized patients compared to outpatient/ED patients for specific ages. For instance, RSV, HRVs, HAdVs, PIVs and $\mathrm{HBoV}$ in young children and HAdVs, PIVs, hMPV and HCoVs in young adults were more frequent in the hospitalized patients. The exception was IFVs, which was more frequent in outpatient/ ED patients (Fig. 1 \& Table 3). Among the hospitalized patients, the viruses detected most frequently were RSV (33.3\%, $n=303 / 1206)$ in children under two years, HAdVs $(13.0 \%, n=42 / 324)$ in patients $15-34$ years, and IFVs $(9.6 \%, n=58 / 602)$ in elderly patients $\geq 65$ years. However, among outpatient/ED patients the most common virus detected was IFVs across all age groups $(22.7 \%, n=1463 / 6437)$.

Viral co-infection was detected in $756(7.6 \%)$ cases, of which 534 (70.6\%) were dual-infection, 150 (19.8\%) were triple-infection, and 72 (9.5\%) were co-infected by more than four different RVs. Patients with more viruses detected were more likely to be hospitalized. Specifically, patients infected by one, two, three, or more than four types of RVs were hospitalized $29.7 \%, 56.0 \%, 68.0 \%$ and $77.8 \%$, respectively $(p<0.001)$. The hospitalized cases generally had more co-infections than outpatient/ED patients $(13.1 \%$ vs. $4.6 \%, \mathrm{p}<0.001)$ (Table 2$)$. Among the hospitalized cases, RSV co-infected with HRVs (RSV:HRVs) was the most common combination identified in children less than five years old $(5.5 \%, n=66$ / 1206) and HAdVs:PIVs in patients five years and older (1.7\%, $n=39 / 2281)$. Among outpatient/ED patients, the most common combinations were IFVs:HRVs in children 
Table 1 Demographic and clinical characteristics of enrolled patients with ARIs in North China, 2012-2015

\begin{tabular}{|c|c|c|c|c|}
\hline \multirow[t]{2}{*}{ Characteristics } & \multirow{2}{*}{$\begin{array}{l}\text { All patients } \\
(n=9924) \text {, no. (\%) }\end{array}$} & \multicolumn{3}{|l|}{ Hospital settings } \\
\hline & & $\begin{array}{l}\text { Outpatient/ED, } \\
(n=6437), \text { no. }(\%)\end{array}$ & $\begin{array}{l}\text { Hospitalized, } \\
(n=3487), \text { no. (\%) }\end{array}$ & $P$-value \\
\hline Male & $5362(54.0)$ & $3329(51.7)$ & $2033(58.3)$ & $<0.001$ \\
\hline Age, median years (IQR) & $25.5(6.6-44.3)$ & $28.2(15.6-42.1)$ & $8.8(3.0-52.0)$ & $<0.001^{*}$ \\
\hline Age in years & & & & $<0.001$ \\
\hline $0-1$ & $1014(10.2)$ & $272(4.2)$ & $742(21.3)$ & \\
\hline $2-4$ & $983(9.9)$ & $519(8.1)$ & $464(13.3)$ & \\
\hline $5-14$ & $1610(16.2)$ & $760(11.8)$ & $850(24.4)$ & \\
\hline $15-34$ & 3070 (30.9) & $2746(42.7)$ & $324(9.3)$ & \\
\hline $35-64$ & $2240(22.6)$ & $1735(27.0)$ & $505(14.5)$ & \\
\hline$\geq 65$ & $1007(10.1)$ & $405(6.3)$ & $602(17.3)$ & \\
\hline Surveillance year & & & & $<0.001$ \\
\hline 2012 & $2517(25.4)$ & $1804(28.0)$ & $713(20.4)$ & \\
\hline 2013 & $2288(23.1)$ & $1201(18.7)$ & $1087(31.2)$ & \\
\hline 2014 & $3198(32.2)$ & $2100(32.6)$ & $1098(31.5)$ & \\
\hline 2015 & $1921(19.4)$ & $1332(20.7)$ & $589(16.9)$ & \\
\hline Season of illness onset ${ }^{a}$ & & & & $<0.001$ \\
\hline winter & $2810(28.3)$ & $1510(23.5)$ & $712(20.4)$ & \\
\hline spring & $2462(24.8)$ & 1789 (27.8) & $1021(29.3)$ & \\
\hline summer & $2222(22.4)$ & $1528(23.7)$ & $934(26.8)$ & \\
\hline autumn & $2430(24.5)$ & $1610(25.0)$ & $820(23.5)$ & \\
\hline Clinical diagnosis & & & & $<0.001$ \\
\hline URTIs ${ }^{b}$ & $5230(52.7)$ & $5069(78.7)$ & $161(4.6)$ & \\
\hline LRT/s c & 4694 (47.3) & $1368(21.3)$ & 3326 (95.4) & \\
\hline Pneumonia ${ }^{d}$ & $4208(42.4)$ & 1134 (17.6) & 3074 (88.2) & \\
\hline Other LRTIs & $486(4.9)$ & 234 (3.6) & $252(7.2)$ & \\
\hline
\end{tabular}

*. Wilcoxon test

a. spring = March to May; summer = June to August; autumn = September to November; winter = December to February

b. URTIs = upper respiratory tract infections, classified when common cold, rhinitis, pharyngitis, laryngitis or otitis media were diagnosed by attending physicians

c. LRTIs = lower respiratory tract infections, classified when pneumonia, bronchiolitis, bronchitis or exacerbations of chronic obstructive pulmonary disease and asthma were diagnosed

d. Pneumonia: chest X-ray showing evidence of consolidation (a dense or fluffy opacity with or without air bronchograms), other infiltrate (linear and patchy alveolar or interstitial densities), or pleural effusion

Table 2 Frequency of respiratory viruses single infection and co-infection among patients with ARIs in North China, 2012-2015, by age group and hospital settings

\begin{tabular}{|c|c|c|c|c|c|c|c|c|c|}
\hline \multirow{2}{*}{$\begin{array}{l}\text { Age } \\
\text { in } \\
\text { years }\end{array}$} & \multicolumn{2}{|c|}{$\begin{array}{l}\text { Single virus infection } \\
(n=3072)\end{array}$} & \multirow[t]{2}{*}{$P$-value } & \multicolumn{2}{|c|}{$\begin{array}{l}\text { Co-infection of viruses } \\
(n=756)\end{array}$} & \multirow[t]{2}{*}{$P$-value } & \multicolumn{2}{|l|}{$\begin{array}{l}\text { Total } \\
(n=3828) \\
\end{array}$} & \multirow[t]{2}{*}{$P$-value } \\
\hline & $\begin{array}{l}\text { Outpatient/ED, } \\
\text { No. (\%) }\end{array}$ & $\begin{array}{l}\text { Hospitalized, } \\
\text { No. (\%) }\end{array}$ & & $\begin{array}{l}\text { Outpatient/ED, } \\
\text { No. (\%) }\end{array}$ & $\begin{array}{l}\text { Hospitalized, } \\
\text { No. (\%) }\end{array}$ & & $\begin{array}{l}\text { Outpatient/ED, } \\
\text { No. (\%) }\end{array}$ & $\begin{array}{l}\text { Hospitalized, } \\
\text { No. (\%) }\end{array}$ & \\
\hline $0-1$ & 105 (38.6) & $278(37.5)$ & 0.77 & $23(8.5)$ & $261(35.2)$ & $<0.001$ & $128(47.1)$ & 539 (72.6) & $<0.001$ \\
\hline $2-4$ & $179(34.5)$ & 148 (31.9) & 0.416 & $27(5.2)$ & $70(15.1)$ & $<0.001$ & $206(39.7)$ & $218(47.0)$ & 0.024 \\
\hline $5-14$ & $245(32.2)$ & 209 (24.6) & $<0.001$ & $44(5.8)$ & $53(6.2)$ & 0.753 & 289 (38.0) & $262(30.8)$ & 0.003 \\
\hline $15-34$ & 896 (32.6) & 66 (20.4) & $<0.001$ & $123(4.5)$ & $31(9.6)$ & $<0.001$ & 1019 (37.1) & 97 (29.9) & 0.012 \\
\hline $35-64$ & $604(34.8)$ & 85 (16.8) & $<0.001$ & $71(4.1)$ & $30(5.9)$ & 0.088 & $675(38.9)$ & 115 (22.8) & $<0.001$ \\
\hline $65+$ & $131(32.3)$ & $126(20.9)$ & $<0.001$ & $11(2.7)$ & $12(2.0)$ & 0.521 & $142(35.1)$ & 138 (22.9) & $<0.001$ \\
\hline Total & 2160 (33.6) & $912(26.2)$ & $<0.001$ & $299(4.6)$ & $457(13.1)$ & $<0.001$ & $2459(38.2)$ & 1369 (39.3) & 0.31 \\
\hline
\end{tabular}




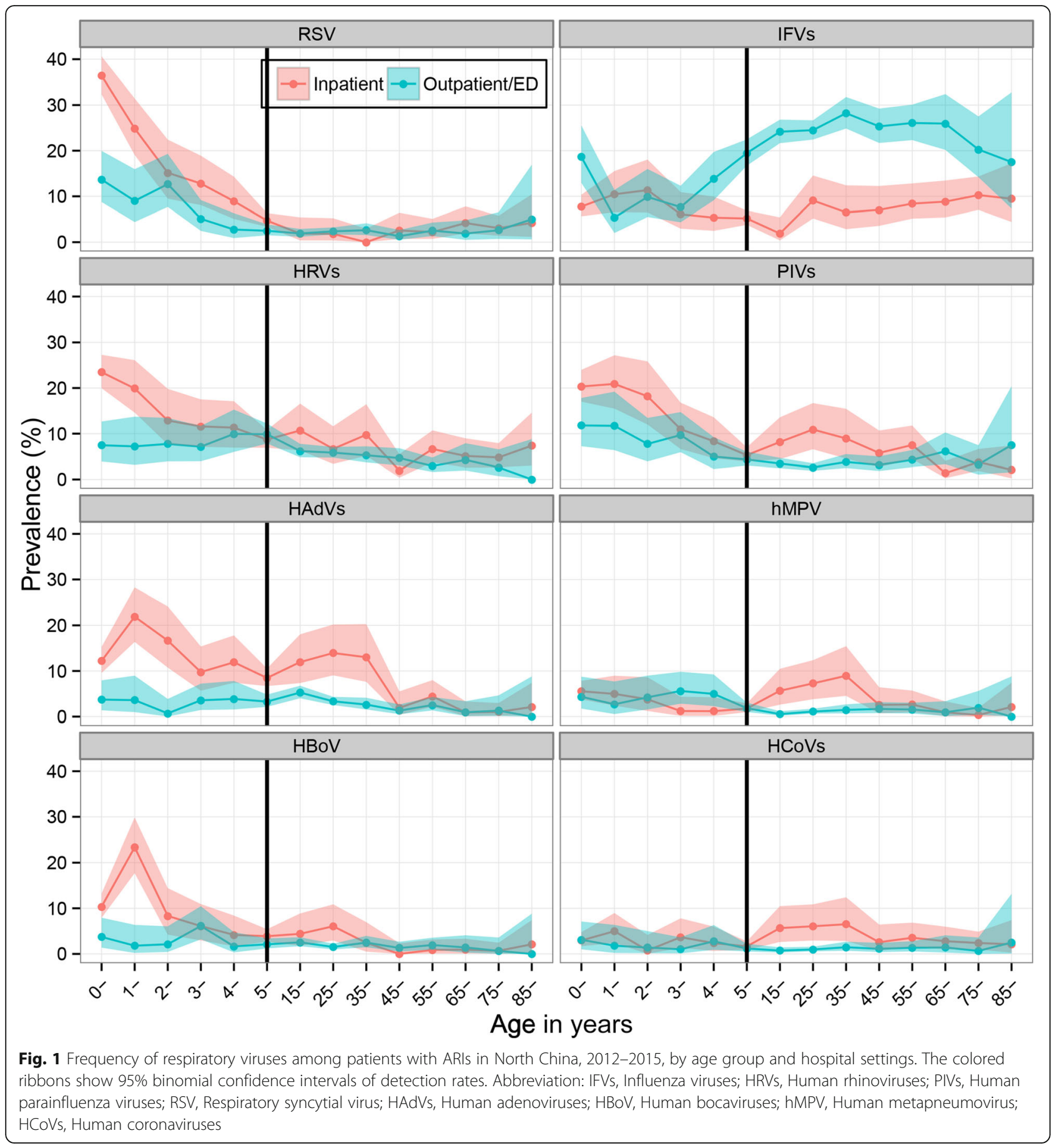

less than five years old $(0.9 \%, n=7 / 791)$, and HAdVs:H$\mathrm{BoV}$ in patients five years and older $(1.2 \%, \mathrm{n}=66 / 5646)$ (Additional file 1).

\section{Risk factors for hospitalization}

All viruses were associated with hospitalization in bivariate analyses $(p<0.001)$ (Additional file 1$)$. However, multivariable analyses revealed that only 3 viruses were consistently associated with hospitalization after adjustment for other viruses and covariates. HAdVs (aOR: 4.03, 99\% CI: 2.21-7.34) and RSV (aOR: 1.72, 99\% CI: 1.11-2.66) as well as RSV co-infected with IFVs (aOR: 12.36, 99\% CI: 2.6358.03) were independently associated with increased hospitalization among children $<14$ years. HAdVs were also associated with hospitalization among adults 15-64 years old (aOR: 2.14, 99\% CI: 1.19-3.85). In contrast, infection of IFVs (aOR: 0.22, 99\% CI: 0.17-0.28) was strongly associated with outpatient/ED patients in all age groups (Fig. 2). 
Table 3 Frequency of respiratory viruses among patients with ARIs in North China, 2012-2015, by age group and hospital settings

\begin{tabular}{|c|c|c|c|c|c|c|c|c|}
\hline \multirow[t]{2}{*}{ Respiratory Viruses } & \multicolumn{4}{|c|}{$\begin{array}{l}\text { Outpatient/ED patients, } \\
\text { No. (\%) }\end{array}$} & \multicolumn{4}{|c|}{$\begin{array}{l}\text { Hospitalized patients, } \\
\text { No. (\%) }\end{array}$} \\
\hline & $\begin{array}{l}\text { Age } 0-14 y, \\
(N=1551)\end{array}$ & $\begin{array}{l}\text { Age } 14-64 \text { y } \\
(N=4481)\end{array}$ & $\begin{array}{l}\text { Age } 65+y \\
(N=405)\end{array}$ & $\begin{array}{l}\text { All } \\
(\mathrm{N}=6437)\end{array}$ & $\begin{array}{l}\text { Age } 0-14 \text { y } \\
(N=2056)\end{array}$ & $\begin{array}{l}\text { Age } 14-64 \text { y } \\
(N=829)\end{array}$ & $\begin{array}{l}\text { Age } 65+y \\
(N=602)\end{array}$ & $\begin{array}{l}\text { All } \\
(\mathrm{N}=3487)\end{array}$ \\
\hline Respiratory syncytial virus & $84(5.4)$ & $98(2.2)$ & $10(2.5)$ & $192(3.0)$ & $343(16.7)$ & $15(1.8)$ & $22(3.7)$ & $380(10.9)$ \\
\hline Influenza viruses & $238(15.3)$ & $1132(25.3)$ & $93(23.0)$ & $1463(22.7)$ & $141(6.9)$ & $56(6.8)$ & $58(9.6)$ & $255(7.3)$ \\
\hline Human rhinoviruses & $138(8.9)$ & $240(5.4)$ & $13(3.2)$ & $391(6.1)$ & $296(14.4)$ & $58(7.0)$ & $32(5.3)$ & $386(11.1)$ \\
\hline Parainfluenza viruses & $104(6.7)$ & $146(3.3)$ & $21(5.2)$ & $271(4.2)$ & $253(12.3)$ & $68(8.2)$ & $16(2.7)$ & $337(9.7)$ \\
\hline Human adenoviruses & $50(3.2)$ & $151(3.4)$ & $4(1.0)$ & $205(3.2)$ & $240(11.7)$ & $71(8.6)$ & $7(1.2)$ & $318(9.1)$ \\
\hline Human metapneumovirus & $50(3.2)$ & $51(1.1)$ & $5(1.2)$ & $106(1.6)$ & $64(3.1)$ & $42(5.1)$ & $5(0.8)$ & $111(3.2)$ \\
\hline Human bocaviruses & $42(2.7)$ & $87(1.9)$ & $4(1.0)$ & $133(2.1)$ & $164(8.0)$ & $22(2.7)$ & $6(1.0)$ & $192(5.5)$ \\
\hline Human coronaviruses & $25(1.6)$ & $47(1.0)$ & $5(1.2)$ & $77(1.2)$ & $51(2.5)$ & $39(4.7)$ & $15(2.5)$ & $105(3.0)$ \\
\hline
\end{tabular}

Age was a strong host predicator of hospitalization. Young children less than two years (aOR: 17.56, 99\% CI: 13.54-22.78) and elderly patients $\geq 65$ years (aOR: 15.78 , 99\% CI: 12.41-20.07) had the highest risk compared with adults aged $15-34$ years. The time of year also revealed environmental risk factors for these groups. For instance, compared to summer, children $<14$ years who were infected during winter (aOR: 1.43, 99\% CI: $1.06-$ 1.91 ) and spring (aOR: 1.42, 99\% CI: $1.08-1.89$ ) were more frequently associated with hospitalization. However, elderly patients who infected during winter (aOR: 1.81, 99\% CI: 1.06-3.08) and autumn (aOR: 2.05, 99\% CI: 1.23-3.39) were more frequently associated with hospitalization (Fig. 2).

\section{Discussion}

Early acquisition of viral diagnosis is helpful for clinicians when treating patients with ARIs by decreasing unnecessary prescription of antibiotics, guiding appropriate use of antiviral agents, and preventing virus transmission in both outpatient and inpatient settings [23]. The wide use of modern molecular techniques enables rapid diagnosis and greatly improves our understanding on the clinical role of respiratory viruses [24]. The high sensitivity of these testing methods reveals multiple pathogens in respiratory specimens (about 12\%-42\% of hospitalized children with ARIs had two or more viruses identified $[5,6,25])$. However, these results were often clinically confusing as it is difficult to distinguish whether the viruses were colonized or prolonged shedding from infection [26]. A better understanding of the distribution of a specific RVs in patients with ARIs in different hospital settings and their interactive relationship with other microbial agents can help clinicians make a better assessment of the role of RVs in the current infections. Toward this end, we simultaneously screened for the presence of eight common respiratory viruses in 9924 patients with ARIs in outpatient and inpatient settings at 11 hospitals in North China. After adjusting for other viruses and significant confounding factors, the detection of RSV, HAdVs, and co-infection of RSV with IFVs was still significantly higher in hospitalized children (or HAdVs in adults) with ARIs than in non-hospitalized children. These data suggest that these infections might predict the severity of ARIs.

RSV is a major viral agent identified in severe LRTIs in young children [27]. In our study, 95.4\% of hospitalized cases were diagnosed with LRTIs, of which one third (34\%) were young children. Of these hospitalized young children, one third (33\%) had RSV detected. These results are highly consistent with previous studies, in which RSV infections were implicated in about $31 \%$ $39 \%$ of hospitalized children $[4,5,28-30]$. In addition, in our study RSV was found more frequently among hospitalized young children than outpatients and was a significant predicator of hospitalization among young children with ARIs. This pattern is consistent with that of previous studies $[5,28]$. Our results confirmed that RSV was still an important health threat to pediatric populations in North China. These data highlight the need to develop effective vaccines and new therapeutics to better treat RSV in North China [31].

IFVs are important causing agents of ARIs [4, 30], and were reported to be the most common identified agent in hospitalized adults $[8,32,33]$. In our study, IFVs was also the most common agent in hospitalized patient $\geq 65$ years. Interestingly, co-infection of RSV with IFVs was a significant predicator of increased hospitalization among young children in our study. Although IFVs were found more frequently among outpatient/ED patients (22.7\%) than among hospitalized cases (7.3\%), 91.1\% of children co-infected with RSV and IFVs were admitted to the hospital. This was significantly higher than children hospitalized with the single infection of RSV (72.2\%, $n=166 / 230)$ or IFVs $(23.8 \%, n=57 / 240)$. Based on these observations, we hypothesized that co-infection of IFVs with other microbial agents, like RSV in our study, might have played an important role in the 


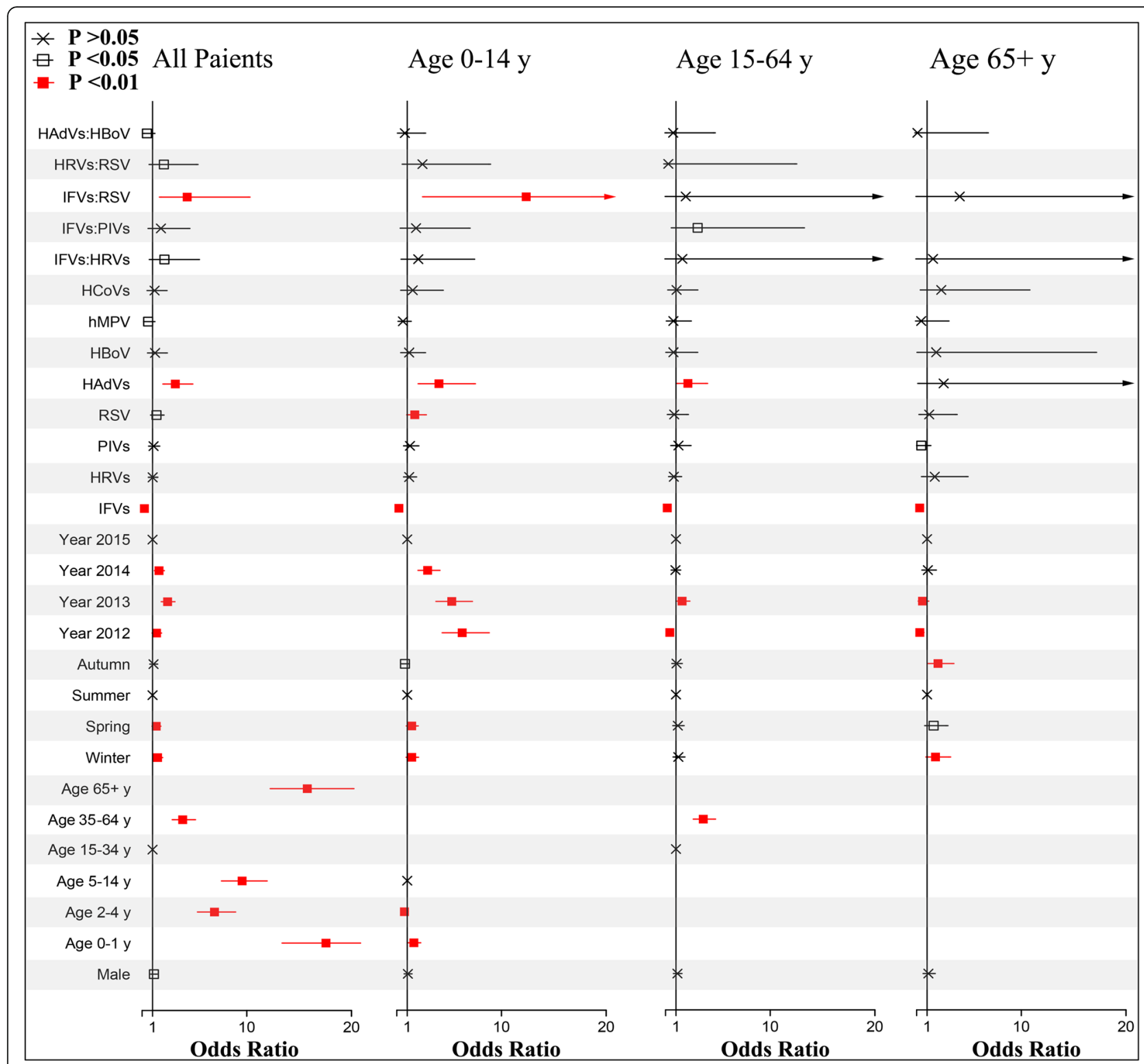

Fig. 2 Adjusted Odds Ratios for risk factors or viruses associated with hospitalization of patients with ARIs, according to age groups. Horizontal lines indicate $99 \%$ confidence intervals. The reference group is assigned aOR = 1. Abbreviation: IFVs, Influenza viruses; HRVs, Human rhinoviruses; PIVs, Human parainfluenza viruses; RSV, Respiratory syncytial virus; HAdVs, Human adenoviruses; HBoV, Human bocaviruses; hMPV, Human metapneumovirus; HCoVs, Human coronaviruses

hospitalization of ARIs in young children as has been described previously [34]. Both RSV and IFVs have caused substantial burden worldwide [7, 35, 36], and the epidemic season of RSV and IFVs are partly overlapping for winter months in China [11]. If our hypothesis that co-infection of IFVs with other microbial agents will cause more severe ARIs among young children is true, the immunization of young children with seasonal influenza vaccine would certainly offer additional benefits by preventing not only IFVs-associated hospitalizations, but also the excessive hospitalizations caused by co-infected RSV or other microbial agents. Since currently no influenza vaccines have been introduced into China's routine immunization program, vaccination of the elderly people and young children with seasonal influenza vaccine should be considered a high priority to reduce the large burden of ARIs among these population groups at high risk of more severe disease.

In our study, viral infection was more common in inpatients than outpatients among younger age group but not older age group. This observation suggests that RVs infection is common among young children and is more likely to be associated with severe ARIs when compared with other age groups, while in adults and elderly 
people, though RVs infection is not uncommon, RVs are less likely to cause severe ARIs in this age group. Moreover, we also found co-infection of two or more viruses in $15 \%-35 \%$ of hospitalized young children as opposed to $2 \%-10 \%$ of hospitalized adults. Higher proportions of co-infection were observed in hospitalized young children than in non-hospitalized children (5\%-9\%). These results suggest that viral co-infection might predict hospitalization of young children but not of adults. Aging is a protecting factor for RVs infections in young children. As the age of children increased, infection and co-infection of RVs became less frequent and were less likely to be associated with hospitalization. This observation suggested that the frequent exposure of children to RVs could lower their risk of RVs-associated infection and/or severe infection, and that the utility of vaccines, when available, could be used to control and prevent the infection of RVs. The most frequent combination of viral co-infection among hospitalized children was RSV with HRVs, which is consistent with that of previous studies [5, 6, 25, 37, 38]. In studies by Papadopoulos et al. and Aberle et al., co-infection of RSV with HRVs was associated with prolonged stay in the hospital in patients with bronchiolitis [25, 37]. In our study, however, the strength of association with hospitalization for RSV co-infected with HRVs was at a marginal value (significant at $p<$ 0.05 but not $p<0.01$ ), which warrants investigation in future studies.

In our study, HAdVs were the most common viral agents among adolescents and young adults hospitalized with ARIs, which is consistent with previous studies [33, 39]. In addition, HAdVs in our study were more frequent in hospitalized children and young adults than in outpatient/ED patients. HAdVs are important agents associated with severe ARIs, for several serotypes of HAdVs (e.g., HAdV-3, 7, 14 and 55), might induce severe and fatal necrotizing pneumonia [26, 40-42]. The host response to HAdVs infection is very similar to that of invasive bacterial infections [43]. In our previous study of the same population in North China, we found that $49 \%$ of identified HAdVs were HAdV-7 [44]. We believe that certain serotypes of HAdVs, e.g. HAdV-7, were more prevalent in this population, which played an important role in the hospitalization of children and young adults in our study. Future studies which sought to sequence the identified HAdVs strains are warranted.

Our study had limitations. First, we did not include an asymptomatic control in our study but rather made comparisons between patient subgroups at different hospital settings which might make the results difficult to interpret. Yet finding no difference does not mean that there is no association of specific virus with the hospitalization of patients. However, the heterogeneous distribution of RVs in patient subgroups at different hospital settings deserved attention from clinicians when treating these patients. Second, we only investigated 2way interactions of viruses. The interactions and bacterial-viral infections were not explored. Third, other host factors were not examined in our study, such as those underlying diseases (e.g. exacerbation of chronic obstructive pulmonary disease, asthma, or cardiovascular diseases), and the previous usage of antibiotics or steroids. The issue of viral-viral co-infection associated with severity of ARIs still remains controversial as laid down by others $[6,45,46]$. Future studies that test multi-pathogens using common standards at multi-sites, employ appropriate community controls and use death as clinical endpoints are warranted.

\section{Conclusions}

In conclusion, a substantial proportion of ARIs are associated with the infection or co-infection of RVs in North China. RSV, HAdVs, and co-infection of RSV and IFVs were more frequent in hospitalized children (or adenoviruses in adults), which might predict the severity of ARIs. Thus, clinicians treating patients with ARIs should be vigilant of these infections. Our findings provide valuable insights into the study of viral pathogenesis and provide guidance for good clinical management of severe ARIs.

\section{Additional file}

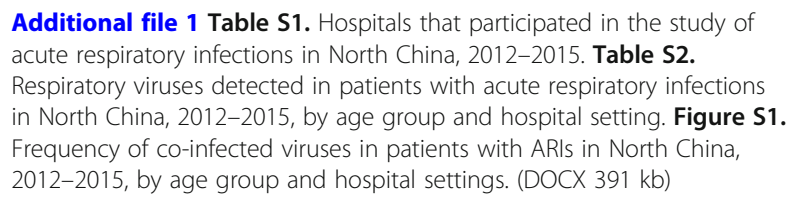

\section{Abbreviations}

95\% Cl: 95\% confidence interval; aOR: adjusted odds ratio; ARIs: Acute respiratory infections; ED: Emergency department; HAdVs: Human adenoviruses; HBoV: Human bocaviruses; HCoVs: Human coronaviruses; hMPV: human metapneumovirus; HRVs: Human rhinoviruses; IFVs: Influenza viruses; LRTIs: Lower respiratory tract infections; PIVs: Parainfluenza viruses; RSV: Respiratory syncytial virus; RT-PCR: Reverse-transcriptase polymerase chain reaction; RVs: Respiratory viruses; URTIs: Upper respiratory tract infections

\section{Acknowledgements}

We wish to thank all the patients, nurses, clinicians, and the laboratory, research, and administrative staff who took part in our study.

\section{Funding}

This work was supported by National Major S \& T Project for the Prevention and Treatment of Major Infectious Diseases in China [2014ZX10004-001, 2017ZX10004206], the Fundamental Research Funds for the Central Universities [2016ZX310060], Program for Changjiang Scholars and Innovative Research Team in University [IRT13007], the funds from postdoctoral science foundation of Peking Union Medical College and Fondation Mérieux. The funders had no role in study design, data collection and analysis, decision to publish, or preparation of the manuscript. 


\section{Availability of data and materials}

The datasets used and/or analyzed during the current study are available from the corresponding authors on reasonable request.

\section{Authors' contributions}

$J Y, Z X, T Z, Y L, H F$ and DY contributed equally to this work. JW, LR, ZX, ZG, $H F, J H$, and TL designed the study. ZX, TZ, YL, HF, DY, KS, FH, JH, TL and ZG collected samples and clinical information, and analyzed the data. JY performed statistical analysis. JY, LR and JW drafted the initial manuscript. LR and JW obtained funding and conceived the study. TB and PV commented and critically revised the manuscript for important intellectual content. All authors read and approved the final manuscript.

\section{Ethics approval and consent to participate}

The study was approved by the ethical review committee at the Institute of Pathogen Biology, Chinese Academy of Medical Sciences (IPB, Beijing, China [Ref. No. IPB-2014-4]). Written consent was obtained before enrollment from each patient or their guardians.

\section{Consent for publication}

Not applicable.

\section{Competing interests}

The authors have no conflicts of interest to disclose.

\section{Publisher's Note}

Springer Nature remains neutral with regard to jurisdictional claims in published maps and institutional affiliations.

\section{Author details}

${ }^{1} \mathrm{MOH}$ Key Laboratory of Systems Biology of Pathogens and Christophe Mérieux Laboratory, IPB, CAMS-Fondation Mérieux, Institute of Pathogen Biology (IPB), Chinese Academy of Medical Sciences (CAMS) \& Peking Union Medical College, Beijing 100730, People's Republic of China. ${ }^{2}$ Key Laboratory of Major Diseases in Children and National Key Discipline of Pediatrics (Capital Medical University), Ministry of Education, Beijing Pediatric Research Institute, Beijing Children's Hospital, Capital Medical University, No. 56 Nan-li-shi Road, Beijing 100045, People's Republic of China. ${ }^{3}$ Beijing Center for Disease Control and Prevention, No.16, Hepingli Middle Avenue of Dongcheng district, Beijing 100013, People's Republic of China. ${ }^{4}$ Shandong Medicinal Biotechnology Centre, Key Laboratory for Modern Medicine and Technology of Shandong Province, Shandong Academy of Medical Sciences, No. 18877 Jingshi Road, Jinan, Shandong 250062, People's Republic of China. ${ }^{5}$ Peking Union Medical College Hospital, Chinese Academy of Medical Sciences \& Peking Union Medical College, Beijing 100730, People's Republic of China. ${ }^{6}$ Department of Respiratory and Critical Care Medicine, Peking University People's Hospital, Beijing 100044, People's Republic of China. 'Service d'Hygiène, Epidémiologie et Prévention, Hôpital Edouard Herriot, Lyon, France. ${ }^{8}$ Laboratoire des Pathogènes Emergents - Fondation Mérieux, Centre International de Recherche en Infectiologie (CIRI), Institut National de la Santé et de la Recherche Médicale U1111, Centre National de la Recherche Scientifique, UMR5308, Ecole Normale Supérieure de Lyon, Université Claude Bernard Lyon 1, 21, Avenue Tony Garnier, 69007 Lyon, France. ${ }^{9}$ INSERM, F-CRIN, I-REIVAC, Lyon Collaborative Center, Lyon, France.

Received: 11 March 2017 Accepted: 30 January 2018

\section{Published online: 08 February 2018}

\section{References}

1. World Health Organization. Research needs for the battle against respiratory viruses (BRaVe). Geneva: WHO Press; 2013. http://www.who.int/influenza/ patient_care/clinical/BRaVe_Research_Agenda_2013.pdf?ua=1.

2. Global Burden of Disease Study Collaborators. Global, regional, and national incidence, prevalence, and years lived with disability for 301 acute and chronic diseases and injuries in 188 countries, 1990-2013: a systematic analysis for the global burden of disease study 2013. Lancet. 2015;386(9995): 743-800.

3. GBD 2013 Mortality and Causes of Death Collaborators: Global, regional, and national age-sex specific all-cause and cause-specific mortality for 240 causes of death, 1990-2013: a systematic analysis for the global burden of disease study 2013. Lancet 2015, 385(9963):117-171.
4. Shi T, McLean K, Campbell H, Nair H. Aetiological role of common respiratory viruses in acute lower respiratory infections in children under five years: a systematic review and meta-analysis. Journal of global health. 2015:5(1):010408.

5. Marcone DN, Ellis A, Videla C, Ekstrom J, Ricarte C, Carballal G, Vidaurreta SM, Echavarria M. Viral etiology of acute respiratory infections in hospitalized and outpatient children in Buenos Aires, Argentina. Pediatr Infect Dis J. 2013;32(3):e105-10.

6. Kouni S, Karakitsos P, Chranioti A, Theodoridou M, Chrousos G, Michos A. Evaluation of viral co-infections in hospitalized and non-hospitalized children with respiratory infections using microarrays. Clin Microbiol Infect. 2013;19(8):772-7.

7. Nair H, Nokes DJ, Gessner BD, Dherani M, Madhi SA, Singleton RJ, O'Brien $\mathrm{KL}$, Roca A, Wright PF, Bruce N, et al. Global burden of acute lower respiratory infections due to respiratory syncytial virus in young children: a systematic review and meta-analysis. Lancet. 2010;375(9725):1545-55.

8. Jain S, Self WH, Wunderink RG, Fakhran S, Balk R, Bramley AM, Reed C, Grijalva CG, Anderson EJ, Courtney DM, et al. Community-acquired pneumonia requiring hospitalization among U.S. adults. N Engl J Med. 2015; 373(5):415-27.

9. Peiris JS, Yuen KY, Osterhaus AD, Stohr K. The severe acute respiratory syndrome. N Engl J Med. 2003;349(25):2431-41.

10. Ren L, Gonzalez R, Wang Z, Xiang Z, Wang Y, Zhou H, Li J, Xiao Y, Yang Q, Zhang J, et al. Prevalence of human respiratory viruses in adults with acute respiratory tract infections in Beijing, 2005-2007. Clin Microbiol Infect. 2009; 15(12):1146-53

11. Feng L, Li Z, Zhao S, Nair H, Lai S, Xu W, Li M, Wu J, Ren L, Liu W, et al. Viral etiologies of hospitalized acute lower respiratory infection patients in China, 2009-2013. PLoS One. 2014;9(6):e99419.

12. Li J, Wang Z, Gonzalez R, Xiao Y, Zhou H, Zhang J, Paranhos-Baccala G, Vernet G, Jin Q, Wang J, et al. Prevalence of human metapneumovirus in adults with acute respiratory tract infection in Beijing, China. The Journal of infection. 2012;64(1):96-103.

13. Ren L, Gonzalez R, Xu J, Xiao Y, Li Y, Zhou H, Li J, Yang Q, Zhang J, Chen L, et al. Prevalence of human coronaviruses in adults with acute respiratory tract infections in Beijing, China. J Med Virol. 2011;83(2):291-7.

14. Xiang Z, Gonzalez R, Wang Z, Xiao Y, Chen L, Li T, Vernet G, ParanhosBaccala G, Jin Q, Wang J. Human rhinoviruses in Chinese adults with acute respiratory tract infection. The Journal of infection. 2010;61(4):289-98.

15. Guo L, Wang Y, Zhou H, Wu C, Song J, Li J, Paranhos-Baccala G, Vernet G, Wang J, Hung T. Differential seroprevalence of human bocavirus species 1-4 in Beijing, China. PLoS One. 2012;7(6):e39644.

16. Xiang Z, Gonzalez R, Ren L, Xiao Y, Chen L, Zhang J, Wang W, Yang Q, Li J, Zhou $\mathrm{H}$, et al. Prevalence and clinical characteristics of human respiratory syncytial virus in Chinese adults with acute respiratory tract infection. J Med Virol. 2013;85(2):348-53.

17. Yu H, Yang W, Varma JK. To save children's lives, China should adopt an initiative to speed introduction of pneumonia vaccines. Health Aff. 2012; 31(11):2545-53

18. Xiang Z, Gonzalez R, Wang Z, Ren L, Xiao Y, Li J, Li Y, Vernet G, ParanhosBaccala G, Jin Q, et al. Coxsackievirus A21, enterovirus 68, and acute respiratory tract infection, China. Emerg Infect Dis. 2012;18(5):821-4.

19. Xiang Z, Tsatsral S, Liu C, Li L, Ren L, Xiao Y, Xie Z, Zhou H, Vernet G, Nymadawa P, et al. Respiratory infection with enterovirus genotype C117, China and Mongolia. Emerg Infect Dis. 2014:20(6):1075-7.

20. Xiang Z, Xie Z, Wang Z, Ren L, Xiao Y, Li L, Vernet G, Paranhos-Baccala G, Shen K, Wang J. Human enterovirus genotype C104, China. Emerg Infect Dis. 2013;19(4):689-91.

21. Ren L, Gonzalez R, Xie Z, Zhang J, Liu C, Li J, Li Y, Wang Z, Kong X, Yao Y, et al. WU and Kl polyomavirus present in the respiratory tract of children, but not in immunocompetent adults. J Clin Virol. 2008;43(3):330-3.

22. R Core Team. R: A language and environment for statistical computing. Vienna: R Foundation for Statistical Computing; 2013. http://www.R-project.org/.

23. Bradley JS, Byington CL, Shah SS, Alverson B, Carter ER, Harrison C, Kaplan SL, Mace SE, GH MC Jr, Moore MR, et al. The management of communityacquired pneumonia in infants and children older than 3 months of age: clinical practice guidelines by the Pediatric Infectious Diseases Society and the Infectious Diseases Society of America. Clin Infect Dis. 2011;53(7):e25-76.

24. Freymuth F, Vabret A, Cuvillon-Nimal D, Simon S, Dina J, Legrand L, Gouarin S, Petitjean J, Eckart P, Brouard J. Comparison of multiplex PCR assays and conventional techniques for the diagnostic of respiratory virus infections in 
children admitted to hospital with an acute respiratory illness. J Med Virol. 2006;78(11):1498-504.

25. Aberle JH, Aberle SW, Pracher E, Hutter HP, Kundi M, Popow-Kraupp T. Single versus dual respiratory virus infections in hospitalized infants: impact on clinical course of disease and interferon-gamma response. Pediatr Infect Dis J. 2005;24(7):605-10.

26. Ruuskanen O, Lahti E, Jennings LC, Murdoch DR. Viral pneumonia. Lancet. 2011;377(9773):1264-75.

27. Hall CB, Weinberg GA, Iwane MK, Blumkin AK, Edwards KM, Staat MA, Auinger P, Griffin MR, Poehling KA, Erdman D, et al. The burden of respiratory syncytial virus infection in young children. N Engl J Med. 2009; 360(6):588-98.

28. Berkley JA, Munywoki P, Ngama M, Kazungu S, Abwao J, Bett A, Lassauniere R, Kresfelder T, Cane PA, Venter M, et al. Viral etiology of severe pneumonia among Kenyan infants and children. JAMA. 2010;303(20):2051-7.

29. Jain S, Williams DJ, Arnold SR, Ampofo K, Bramley AM, Reed C, Stockmann C, Anderson EJ, Grijalva CG, Self WH, et al. Community-acquired pneumonia requiring hospitalization among U.S. children. N Engl J Med. 2015;372(9): 835-45.

30. Yoshida LM, Suzuki M, Nguyen HA, Le MN, Dinh Vu T, Yoshino H, Schmidt WP, Nguyen TT, Le HT, Morimoto K, et al. Respiratory syncytial virus: coinfection and paediatric lower respiratory tract infections. Eur Respir J. 2013; 42(2):461-9.

31. Mazur NI, Martinon-Torres F, Baraldi E, Fauroux B, Greenough A, Heikkinen T, Manzoni P, Mejias A, Nair H, Papadopoulos NG, et al. Lower respiratory tract infection caused by respiratory syncytial virus: current management and new therapeutics. Lancet Respir Med. 2015;3(11):888-900.

32. Thompson WW, Shay DK, Weintraub E, Brammer L, Bridges CB, Cox NJ Fukuda K. Influenza-associated hospitalizations in the United States. JAMA. 2004;292(11):1333-40.

33. Qu JX, Gu L, Pu ZH, Yu XM, Liu YM, Li R, Wang YM, Cao B, Wang C. Beijing Network for Adult Community-Acquired P: Viral etiology of communityacquired pneumonia among adolescents and adults with mild or moderate severity and its relation to age and severity. BMC Infect Dis. 2015;15:89.

34. Mina MJ, Klugman KP. The role of influenza in the severity and transmission of respiratory bacterial disease. Lancet Respir Med. 2014;2(9):750-63.

35. Nair H, Brooks WA, Katz M, Roca A, Berkley JA, Madhi SA, Simmerman JM, Gordon A, Sato M, Howie S, et al. Global burden of respiratory infections due to seasonal influenza in young children: a systematic review and metaanalysis. Lancet. 2011;378(9807):1917-30.

36. Poehling KA, Edwards KM, Weinberg GA, Szilagyi P, Staat MA, Iwane MK, Bridges CB, Grijalva CG, Zhu Y, Bernstein Dl, et al. The underrecognized burden of influenza in young children. N Engl J Med. 2006;355(1):31-40.

37. Papadopoulos NG, Moustaki M, Tsolia M, Bossios A, Astra E, Prezerakou A, Gourgiotis D, Kafetzis D. Association of rhinovirus infection with increased disease severity in acute bronchiolitis. Am J Respir Crit Care Med. 2002; 165(9):1285-9.

38. Richard N, Komurian-Pradel F, Javouhey E, Perret M, Rajoharison A, Bagnaud A, Billaud G, Vernet $G$, Lina B, Floret $D$, et al. The impact of dual viral infection in infants admitted to a pediatric intensive care unit associated with severe bronchiolitis. Pediatr Infect Dis J. 2008; 27(3):213-7.

39. Guo L, Gonzalez R, Zhou H, Wu C, Vernet G, Wang Z, Wang J. Detection of three human adenovirus species in adults with acute respiratory infection in China. Eur J Clin Microbiol Infect Dis. 2012;31(6):1051-8.

40. Tang L, Wang L, Tan X, Xu W. Adenovirus serotype 7 associated with a severe lower respiratory tract disease outbreak in infants in Shaanxi Province, China. Virol J. 2011;8:23.

41. Sun B, He H, Wang Z, Qu J, Li X, Ban C, Wan J, Cao B, Tong Z, Wang C Emergent severe acute respiratory distress syndrome caused by adenovirus type 55 in immunocompetent adults in 2013: a prospective observational study. Crit Care. 2014;18(4):456.

42. Chen Y, Liu F, Wang C, Zhao M, Deng L, Zhong J, Zhang Y, Ye J, Jing S, Cheng $Z$, et al. Molecular identification and epidemiological features of human adenoviruses associated with acute respiratory infections in hospitalized children in southern China, 2012-2013. PLoS One. 2016;11(5): e0155412.

43. British Thoracic Society of Standards of Care Committee. BTS quidelines for the management of community acquired pneumonia in childhood. Thorax. 2002;57(suppl 1):i1-i24
44. Liu C, Xiao Y, Zhang J, Ren L, Li J, Xie Z, Xu B, Yang Y, Qian S, Wang J, et al. Adenovirus infection in children with acute lower respiratory tract infections in Beijing, China, 2007 to 2012. BMC Infect Dis. 2015;15:408.

45. Cebey-Lopez M, Herberg J, Pardo-Seco J, Gomez-Carballa A, Martinon-Torres N, Salas A, Martinon-Sanchez JM, Justicia A, Rivero-Calle I, Sumner E, et al. Does viral co-infection influence the severity of acute respiratory infection in children? PLoS One. 2016;11(4):e0152481.

46. Asner SA, Science ME, Tran D, Smieja M, Merglen A, Mertz D. Clinical disease severity of respiratory viral co-infection versus single viral infection: a systematic review and meta-analysis. PLoS One. 2014;9(6):e99392.

\section{Submit your next manuscript to BioMed Central and we will help you at every step:}

- We accept pre-submission inquiries

- Our selector tool helps you to find the most relevant journal

- We provide round the clock customer support

- Convenient online submission

- Thorough peer review

- Inclusion in PubMed and all major indexing services

- Maximum visibility for your research

Submit your manuscript at www.biomedcentral.com/submit
Biomed Central 